\title{
Comparing the effectiveness of eye movement desensitization reprocessing and cognitive behavioral therapy in reducing post-traumatic stress disorder
}

\section{BACKGROUND}

Post-traumatic stress disorder (PTSD) is prevalent in children, adolescents and adults. It can occur alone or in comorbidity with other disorders. A broad range of psychotherapies such as cognitive behavioral therapy (CBT) and eye movement desensitization reprocessing (EMDR) have been developed for the treatment of PTSD. The aim of this study was to compare the effectiveness of eye movement desensitization reprocessing and cognitive behavioral therapy in reducing post-traumatic stress disorder.

\section{PARTICIPANTS AND PROCEDURE}

The population of this investigation was composed of 450 veterans of holy war who referred to military health services. The study sample contained 225 veterans who were randomly selected and asked to complete the Mississippi Post Traumatic Stress Disorder Questionnaire. From 80 respondents who achieved above the minimum score in the questionnaire, 40 persons were chosen randomly and assigned to two experimental groups and one control group. Subjects in the experimental group received eight sessions of CBT training and 8 sessions of EMDR therapy. Post tests were taken from all participants after therapy sessions. Data were analyzed using ANCOVA in SPSS version 25.

RESULTS

Our findings indicated a significant difference between EMDR and CBT groups in term of average PTSD. We also found a significant difference in terms of average PTSD between the control group and CBT as well as EMDR groups.

\section{CONCLUSIONS}

The results of the present study indicated that the eye movement desensitization reprocessing method and cognitive behavioral therapy are effective in reducing post-traumatic stress disorder. We also found that EMDR is more effective than CBT.

KEY WORDS

cognitive behavioral therapy; eye movement desensitization reprocessing; post-traumatic stress disorder 


\section{BACKGROUND}

The last subtype of anxiety disorders is post-traumatic stress disorder (PTSD). In this disorder, psychosomatic symptoms occur following a traumatic psychological event that is usually beyond the human experience. The triggers that cause this syndrome in most people cause symptoms of distress (Rosenhan \& Seligman, 1995). The patient's reactions to these events included severe fear, helplessness, or panic.

Sahar Aligholizadeh Moghadam, Reza Kazemi, Somayyeh Taklavi

Traumatic events are continually re-experienced by one of the following: events such as mental images, thoughts, or per-
1. Repeated and annoying reminders of distressing ceptions.

2. The person's action or feeling is as if the event is being repeated (including feelings of renewal, sensory errors, delusions, and delayed reaction times, including those that may be awakening or poisoning).

3. Severe psychological distress when confronted with internal or external symptoms that are symbolic or similar to an aspect of a traumatic event.

4. Physiological reactivity in the face of internal or external symptoms that symbolize or resemble an aspect of a traumatic event. A person usually makes a conscious effort to avoid thoughts, feelings, or conversations about the traumatic event and to avoid activities, situations, or people that evoke memories of that event. This avoidance of reminders may include remembrance of an important aspect of a traumatic event, a marked decrease in interest or participation in important activities, a feeling of disgust or dislike of others, a limitation of the range of emotions, and a feeling of future shortening. The disorder lasts longer than a month. This disorder results in major discomfort or clinical disturbance in social, occupational, or other important areas. It is acute if the symptoms last less than three months and chronic if they last three months or more. If the onset of symptoms is at least six months after the traumatic event occurs, the type is delayed (Rosenhan \& Seligman, 1995).

Post-traumatic stress disorder is a type of anxiety in people who have experienced an accident, such as the military community and veterans of the imposed war. PTSD can follow experiences such as threats, fears or unpleasant news, or events such as war, natural disasters, severe accidents, and the occurrence of adverse events. It is associated with impairment of one's ability to work, community, acquaintances and family, including job instability, marital problems, difficulties in parenting, divorce, and so on. Stressful events are a major part of the lives of veterans. Having a physical defect, treating it and being constantly involved with any of these problems has a range of stress for these people. But while the cognitive vulnerability precedes the stressful event (here the physical or psychological defect of the war veterans) because many of them have not experienced proper treatment due to stress and stress, therefore, according to research, cognitive therapy can play a significant role in ameliorating the symptoms of veterans. In Iran also there are various ways to treat PTSD (O’Doherty et al., 2017).

The method of treatment of eye movement desensitization reprocessing (EMDR) has been recognized in recent years as one of the effective ways to reduce the symptoms of PTSD. This method was developed by Lang (1977) and Bower (1981), and is based on the information processing model. Eye movement desensitization reprocessing is a form of exposure therapy for clinicians who have experienced traumatic stress. This treatment includes visual immersion, cognitive remodeling, and the rapid and balanced use of eye movements and stimulation of the other two. The treatment that Shapiro invented came from behavioral interventions and was designed to help clinicians with post-traumatic stress disorder. This method has so far been applied to diverse populations, including children, couples, accident victims, people who are anxious, frightened, depressed, sad, addicted, and those with phobias (Shapiro, 1994).

The treatment of eye movement desensitization reprocessing may also be effective for anxiety related to bold behavior. Researchers say treatment with eye movement desensitization reprocessing helps people overcome the feelings of guilt, fear, discomfort, defective thinking, and anxiety that typically result from traumatic experiences (Gorman et al., 1989).

Cognitive behavioral therapy is the result of the developmental course of behavioral therapy. In the UK, Michael Golder has been a pioneer in behavioral therapy. He and his colleagues made Maudsley Hospital a place to conduct research on behavioral therapies. In the field of behavioral psychology (which is different from behavioral therapy), the importance of cognitive variables has been increasingly emphasized. Perhaps the reason for the slow pace of cognitive behavioral assimilation by therapeutic behavior is related to Watson's continued influence on inpatient rejection as well as the attitudes of therapists to other psychotherapy modalities (Paunović, 2010).

Integrating the traditional (behavioral therapy) with the new method (cognitive therapy) was not without problems and we have now realized that behavioral change is more achievable and measurable than cognitive change (which is unstable and fluctuating). Researchers and therapists are now more interested in explanations, understandings, aspirations and fears of patients (clients) than ever before, and coordinate with them. Cognitive therapy provides the context for behavioral therapy. This evolution reflects the relationship between phenomenological 
and cognitive therapy. A series of chain processes during cognitive behavioral therapy sessions results in a time point between sessions; that is, cognitive behavioral therapy sessions are the key to initiating emotional processing. This processing is completed in the interval between sessions (Wright, Brown, Thase, \& Basco, 2017).

The results of a study conducted to evaluate the effectiveness of cognitive behavioral therapy on the mental health of post-traumatic stress disorder veterans showed that cognitive behavioral therapy is an effective clinical intervention in ameliorating symptoms of veterans with post-traumatic stress disorder, and it can help improve their mental health and quality of life (Nixon \& Bralo, 2019). The results of another study conducted to evaluate the effectiveness of cognitive behavioral therapy (CBT) in patients with post-traumatic stress disorder show that cognitive behavioral therapy is effective in reducing the symptoms of PTSD patients (Padmanabhanunni \& Edwards, 2016). Another study conducted in 2017 showed that the two groups were significantly different in performance in the two Stroop tests (Scott et al., 2017).

Another study comparing motor ocular desensitization with cognitive behavioral therapy in the treatment of stress disorder showed that although both EMDR and CBT were effective in the treatment of PTSD, EMDR is more effective in reducing PTSD symptoms than Iranian CBT (Carlson, Chemtob, Rusnak, Hedlund, \& Muraoka, 1998). Another study, conducted as a long-term course of post-traumatic stress disorder PTSD in German soldiers, to investigate the effects of desensitizing the patient's eye movement and refining treatment and traumaspecific features in patients with non-combat PTSD, found that these patients were significantly improved by EMDR therapy. Researchers in a study using EMDR to treat patients with PTSD have shown that this type of treatment is effective in improving many of the symptoms of the disease that can disrupt the activities of daily living (Sadatmadani, Tavallaii Zavareh, \& Tavallaii, 2018). In a study of Syrian refugees with PTSD, Acarturk et al. (2015) found that EMDR significantly reduces symptoms of this disorder. In addition, Hagen Smid, Knipscheer, and Kleber (2015) in a review of treatments for PTSD found that EMDR treatment had no negative effects after treatment in combat casualties and reduced symptoms of the disorder.

Given the importance of the subject and what was said, this study seeks to compare the effectiveness of cognitive behavioral therapy and eye movement desensitization reprocessing in reducing symptoms of post-traumatic stress disorder, and whether cognitive behavioral therapy and eye movement desensitization reprocessing are effective in reducing symptoms of post-traumatic stress disorder.

\section{PARTICIPANTS AND PROCEDURE}

This research was done using an experimental method. The research design is pre-test-post-test with a control group. The study population consisted of all patients with PTSD referred to Isar Hospital in Ardabil in 2019. Using the random sampling method, among the people who scored above 50 on the PCL questionnaire, forty-five participants were selected and randomly assigned to 2 experimental groups $(n=15)$ and a control group $(n=15)$. One group underwent 8 sessions of cognitive behavioral therapy and the other group underwent 4 sessions of postprocessing eye tracking repetition. After treatment sessions, the post-test was taken from all participants.

Data were analyzed using ANCOVA and SPSS 25 software.

\section{CBT TREATMENT METHOD}

Stages and implementation process of CBT treatment method:

First sessions: Introducing the treatment and implemented arrangements.

Second sessions: 1 - Focus on skill training of problem solving, 2 - Definition and classification of formula and production of alternative solutions.

Third sessions: Finding the value of the produced solution.

Fourth sessions: Decision making.

Fifth sessions: Proving and reviewing the process of problem solving.

Sixth sessions: Investigating the problems of each patient based on the process of problem solving.

Seventh sessions: Emphasizing personal problems, definitions and classification of formula.

Eighth sessions: Finding the value of the presented solutions.

Ninth sessions: Finding the value of the presented solutions.

Tenth sessions: Proof stage.

Eleventh sessions: End of the treatment.

\section{EMDR TREATMENT METHOD}

Implementation of procedures and processes of EMDR treatment method:

First sessions: Introducing treatment and preliminary measures of the planning:

1. Introduction of the therapist and patients and providing relation.

2. Brief introduction of treatment.

3. Expressing the expectations and the way of patients' participation in treatment by the therapist.

4. Implementation of the pre-test questionnaires.

5. Getting the patient records and making them ready.
Comparing the effectiveness of EMDR and CBT on reducing $P T S D$ 
Second sessions: Evaluation:

1. Introduction of the therapist and patients and providing relation.

2. Brief introduction of treatment.

3. Expressing the expectations and way of patients' participation in treatment by the therapist.

4. Implementation of the pre-test questionnaires.

5. Getting the patient records and making them ready. Third sessions: Cognitive reconstruction:

1. Applying.

Sahar

Aligholizadeh Moghadam, Reza Kazemi, Somayyeh Taklavi

2. Surveying and paying attention to physical problems.

Fourth sessions: Evaluation of the treatment process:

1. Final step.

2. Re-evaluation.
3. End of the treatment process.

4. Implementation of post-test and evaluation of patients' status.

\section{RESEARCH TOOLS}

Mississippi (Echelle) PTSD measure. This questionnaire has been developed. The items have been manipulated based on the Iranian culture in 2004 and included 39 items scored from 1 to 5 according to the Likert scale. The total score range was from 39 to 195 . Cronbach's $\alpha$ coefficient was reported as .92 and test-retest reliability over a week was .91 . Furthermore, concurrent validity of the questionnaire was measured via its correlation with the PTSD symptom list, and the resulting coefficient was .82 (Goodarzi, 2003).

\section{RESULTS}

In order to test the hypotheses, the mean scores of pre-test and post-test differences in the two groups of experimental and control groups in the stress disorder variable were evaluated by ANCOVA.

- There is a linear relationship between the random auxiliary variable and the dependent variable.

- Slope homogeneity assumption (regression).

- Assumption of variance equality.

- The analysis of covariance analysis showed that the covariance analysis test can be used.

The results of Tables 1 and 2 show that there is a significant difference between the mean post-traumatic stress disorder post-test of the three control groups, EMDR and CBT.

1. There was a significant difference $(p<.01)$ between the mean of post-traumatic stress disorder (EMDR) group and the mean of post-traumatic stress disorder (CBT) group. This difference is for the benefit of the EMDR group.

2. There was a significant difference $(p<.01)$ between the mean post-traumatic stress disorder in the control group and the mean post-traumatic stress disorder in the EMDR group. This difference is for the benefit of the EMDR group.

3. There was a significant difference $(p<.01)$ between the mean of post-traumatic stress disorder in the control group and the mean of post-traumatic stress disorder in the CBT group. This difference is for the benefit of the CBT group.

Thus it can be concluded that both EMDR and CBT training methods are effective in post-traumatic stress

Table 1

Mean and standard deviation of study groups in pre-test and post-test according to stress disorder and paired comparison of two groups with Tukey post hoc test

\begin{tabular}{lcrrrrrr}
\hline \multirow{2}{*}{ Row } & \multirow{2}{*}{ Groups } & \multicolumn{2}{c}{ Pre-test } & \multicolumn{2}{c}{ Post-test } & HSD Tukey & $p$ \\
\cline { 3 - 6 } & & $M$ & $S D$ & $M$ & $S D$ & test & \\
\hline 1 & EMDR & 22.10 & 9.08 & 20.44 & 6.10 & $2>1$ & $<.001$ \\
2 & CBT & 5.86 & 26.93 & 8.86 & 26.46 & $3>2$ & $<.001$ \\
3 & Control & 11.81 & 33.67 & 12.81 & 32.44 & $3>1$ & $<.001$ \\
\hline
\end{tabular}

Table 2

Summary of analysis of covariance of stress disorder in experimental and control groups by interaction effect

\begin{tabular}{lccccccc}
\hline Variable & Source & Sum of squares & $d f$ & Mean of squares & $F$ & $p$ & $\eta$ \\
\hline Stress & Pre-test & 4271.47 & 1 & 4271.46 & 652.50 & $<.001$ & .94 \\
disorder & Between groups & 798.18 & 2 & 399.09 & 60.96 & $<.001$ & .75 \\
& Within groups & 268.38 & 41 & 6.54 & & & \\
& Total & 38665 & 45 & & & \\
\hline
\end{tabular}


disorder and, in comparison with each other, the effectiveness of the EMDR method was greater than the CBT method with respect to the effect size $(\eta=.94)$.

The data in Table 2 show that the $F$ ratio for the effect of application is statistically significant $(\eta=.75$, $p<.001, F=60.96$ (2 \& 41)). This shows that there is a difference between the three groups. In other words, there is a significant difference between the two experimental methods in reducing stress disorder with the control group. Since $F$ is statistically significant, follow-up tests should be used. For this purpose, a pairwise comparison test called a custom hypothesis was run with the Lmatrix command. Tukey's test was used to determine the significance level of these tests.

\section{DISCUSSION AND CONCLUSIONS}

According to the findings both EMDR and CBT training methods are effective in reducing post-traumatic stress disorder symptoms and the effectiveness of the EMDR method is greater than that of the CBT method. These results are in line with the findings of Jeffries and Davis (2013) and Narimani and Rajabi (2009). In explaining this finding we can make a few points in analyzing the results and effectiveness of EMDR.

This treatment accelerates the access, retrieval and reprocessing of traumatic memories in a consistent fashion (Shapiro \& Solomon, 2010) and it helps the brain with natural processing of emotional information.

To be released from traumatic nervous system trauma (Shapiro, 1998), in examining EMDR-related theories, it can be argued that this effect may be due to the activation of the nervous system and memory and emotional networks - which occur through the stimulation of the eye in the context of an orderly process. It has a pervasive effect on facilitating the processing of information from disturbing memories, and because veterans with this disorder suffer from three physiological, behavioral, and cognitive aspects, they have problems and symptoms that indicate their incompatibility in family, occupational, and social settings, and since cognitive behavioral therapy focuses on the cognitive and behavioral dimension of veterans and the therapeutic approach of rapid eye movements and reprocessing of most physiological parts and the remake of the past (Flashback) targets the remnants of war events.

Continuous and regular treatment sessions were performed according to the two treatment packages. According to reports and follow-up after treatment of veterans and their families, two therapies seem to be effective in removing symptoms of the disorder and making them more socially behaviorally compatible. There was also a significant difference between the mean of post-traumatic stress disorder and the mean of EMDR post-traumatic stress disorder. In this way, the treatment through the eye movement desensitization reprocessing has an effect on reducing the symptoms of traumatic stress disorder. The findings also suggest that EMDR treatment can help patients with post-traumatic stress disorder such as wars, floods, earthquakes, driving accidents, bombings, horrors or living in captives' camps to produce favorable results and patients with PTSD after EMDR treatment sessions showed a significant decrease in symptoms of post-traumatic stress disorder (Lee, Gavriel, Drummond, Richards, \& Greenwald, 2002).

These results are consistent with the findings of Sadatmadani et al. (2018), Acarturk et al. (2015), Haagen et al. (2015), Zimmermann, Biesold, Barre, and Lanczik (2007), Ironson, Freund, Strauss, and Williams (2002), Carlson et al. (1998), Shapiro (1996), Ashaiery, Hooman, Jamali, and Vatankhah (2009).

In explaining this finding, it can be said that in the EMDR method, the emphasis has been on facilitating the therapeutic effects of the brain's self-healing mechanism by disrupting previous connections and disassociating the neurophysiological networks of the relevant memory. In addition, due to the physical distance between the small networks of neural connections, the success of linking between them occurs rapidly. Therefore, its therapeutic results do not take long, and occur in a limited time. This is in accordance with Lang's theory of fast information processing. Indeed, the rapid occurrence of the therapeutic effects of EMDR on the findings of this study is largely justified. Also, in the EMDR method, patients are encouraged to pay attention to the details of negative thoughts and make no effort to guide this process. Such a situation creates and uses a dynamic and fluid state of re-exposure compared to other exposurebased methods, by re-invoking emotional responses to various stimuli related to negative thoughts. Such fluidity and dynamics eventually lead to the occurrence of Pavlovian offsets in non-adaptive behaviors (Renfrey \& Spates, 1994).

Another result was that there was a significant difference between the mean of post-traumatic stress disorder in the control group and the mean of post-traumatic stress disorder in the CBT group. This means that cognitive behavioral therapy has the effect of reducing the symptoms of traumatic stress disorder. Cognitive behavioral therapy is based on the idea that psychological problems arise from our interpretation or evaluation of our situations, thoughts, and feelings, and behaviors; so the purpose of CBT is to help people through training in coping with distressing thoughts as well as reducing avoidance or other problematic behaviors. Ideally, if you can change someone, that change should be about evaluating his or her thoughts and environment (Matthews \& Williams, 2008). CBT is used
Comparing the effectiveness of EMDR and CBT on reducing $P T S D$ 
to successfully treat a range of problems, such as anxiety disorders, depression, eating disorders, alcoholism, and drug abuse (Barlow, Gorman, Shear, \& Woods, 2000). These results are consistent with the findings of Nixon and Bralo (2019), Padmanabhanunni and Edwards (2016), Mirdoraghi, Hashemabady, and Mashhadi (2012), Beck, Coffey, Foy, Keane, and Blanchard (2009), Aslani, Hashemiyan, Lotfi Kashani, and Mirzayi (2007), Rothbaum et al. (2006), Mahmoudi-Gharaei, Mohammadi, Bina, Yasami, and Sahar Fakour (2006), Resick, Nishith, Weaver, Astin, and Aligholizadeh Moghadam, Reza Kazemi, Somayyeh Taklavi Feuer (2002).

In explaining the results, it can be said that the cognitive behavioral approach emphasizes that the concepts are operationalized and validate treatment empirically. In this type of treatment, the patient is helped to identify distorted patterns of thought and to identify their dysfunctional behavior. Regular discussions and precisely organized behavioral tasks are used to overcome these distorted thoughts and change their dysfunctional behavior. In some aspects of treatment, emphasis is mainly on behavioral and on other cognitive aspects. Much of the treatment is based on the approach here and now, and the main purpose of treatment is to help the patient make the desired changes in their lives. Therefore, the emphasis on treatment is to provide opportunities for new adaptive learning as well as to make changes outside the clinical context. Problem solving is an important component of treatment. All aspects of the treatment are explained to the patient and the therapist and the patient try to interact and develop strategies to deal with precisely identified issues. This approach is mainly based on self-help and the goal of the therapist is to help the patient develop the skills needed to solve not only current but similar problems in the future. The therapist should emphasize the role of homework and keep in mind that much of the treatment is done in everyday life. This means that the patient has everything discussed in the treatment sessions, and brings it into action. The collaborative nature of therapeutic communication should be discussed. The patient is expected to actively participate in treatment through gathering information, providing feedback on therapeutic techniques, and proposing new strategies. Information on the structure of treatment should also be given to the patient at this stage, such as the number of treatment sessions and the location of the sessions and the duration of treatment (Shapiro, 1998).

\section{References}

Acarturk, C., Konuk, E., Cetinkaya, M., Senay, I., Sijbrandij, M., Cuijpers, P., \& Aker, T. (2015). EMDR for Syrian refugees with posttraumatic stress disorder symptoms: Results of a pilot randomized controlled trial. European Journal of Psychotraumatology, 6, 27414. https://doi.org/10.3402/ejpt.v6.27414

Ashaiery, H., Hooman, H. A., Jamali, M., \& Vatankhah, H. R. (2009). The compares effects of eye movement desensitization and reprocessing, medical, cognitive therapies approaches on decreasing the state anxiety. Journal of Psychological Researches, 1, 51-63.

Aslani, M., Hashemiyan, K., Lotfi Kashani, F., \& Mirzayi, J. (2007). The effectiveness of cognitive behavior therapy on chronic post-traumatic stress disorder patients. Applied Psychology, 1, 6-17.

Barlow, D. H., Gorman, J. M., Shear, M. K., \& Woods, S. W. (2000). Cognitive-behavioral therapy, imipramine, or their combination for panic disorder: a randomized controlled trial. Journal of American Medical Association, 283, 2529-2536. https://doi.org/10.1001/jama.283.19.2529

Beck, J. G., Coffey, S. F., Foy, D. W., Keane, T. M., \& Blanchard, E. B. (2009). Group cognitive behavior therapy for chronic posttraumatic stress disorder: an initial randomized pilot study. Behavior Therapy, 40, 82-92. https://doi.org/10.1016/j. beth.2008.01.003

Bower, G. H. (1981). Mood and memory. American Psychologist, 36, 129-148. https://doi.org/10.1037/0003066X.36.2.129

Carlson, J. G., Chemtob, C. M., Rusnak, K., Hedlund, N. L., \& Muraoka, M. Y. (1998). Eye movement desensitization and reprocessing (EMDR) treatment for combat-related posttraumatic stress disorder. Journal of Traumatic Stress, 11, 3-24. https://doi.org/10.1023/A:1024448814268

Goodarzi, M. A. (2003). Evaluating reliability and validity of the Mississippi scale for post-traumatic stress disorder in Shiraz. Journal of Psychology, 26, 152-178.

Gorman, J. M., Battista, D., Goetz, R. R., Dillon, D. J., Liebowitz, M. R., Fyer, A. J., \& Klein, D. F. (1989). A comparison of sodium bicarbonate and sodium lactate infusion in the induction of panic attacks. Archives of General Psychiatry, 46, 145-150. https:// doi.org/10.1001/archpsyc. 1989.01810020047008

Haagen, J. F., Smid, G. E., Knipscheer, J. W., \& Kleber, R. J. (2015). The efficacy of recommended treatments for veterans with PTSD: a metaregression analysis. Clinical Psychology Review, 40, 184-194. https://doi.org/10.1016/j.cpr.2015.06.008

Ironson, G., Freund, B., Strauss, J. L., \& Williams, J. (2002). Comparison of two treatments for traumatic stress: A community-based study of EMDR and prolonged exposure. Journal of Clinical Psychology, 58, 113-128. https://doi.org/10.1002/jclp.1132

Jeffries, F. W., \& Davis, P. (2013). What is the role of eye movements in eye movement desensitization and reprocessing (EMDR) for post-traumatic stress disorder (PTSD)? A review. Behavioural and Cognitive Psychotherapy, 41, 290-300. https://doi. org/10.1017/S1352465812000793 
Lang, P. J. (1977). Imagery in therapy: an information processing analysis of fear. Behavior Therapy, 8, 862886. https://doi.org/10.1016/S0005-7894(77)80157-3

Lee, C., Gavriel, H., Drummond, P., Richards, J., \& Greenwald, R. (2002). Treatment of PTSD: Stress inoculation training with prolonged exposure compared to EMDR. Journal of Clinical Psychology, 58, 1071-1089. https://doi.org/10.1002/jclp.10039

Mahmoudi-Gharaei, J., Mohammadi, M. R., Bina, M., Yasami, M. T., \& Fakour, Y. (2006). Supportive and cognitive behavioral group interventions on Bam earthquake related PTSD symptoms in adolescents. Tehran University Medical Journal, 64, 57-67.

Matthews, M. M., \& Williams, H. G. (2008). Can Tai chi enhance cognitive vitality? A preliminary study of cognitive executive control in older adults after a Tai chi intervention. Journal of the South Carolina Medical Association, 104, 255-257.

Mirdoraghi, F., Hashemabady, G., \& Mashhadi, A. (2012). Cognitive and behavioral inhibition in veterans with-and without post-traumatic stress disorder. Journal of Military Medicine, 14, 41-47.

Narimani, M., \& Rajabi, S. (2009). Comparison of efficacy of eye movement, desensitization and reprocessing and cognitive behavioral therapy methods in the treatment of stress disorder. Medical Science Journal of Islamic Azad University, 19, 236-245.

Nixon, R. D., \& Bralo, D. (2019). Using explicit case formulation to improve cognitive processing therapy for PTSD. Behavior Therapy, 50, 155-164. https://doi.org/10.1016/j.beth.2018.04.003

O’Doherty, D. C., Tickell, A., Ryder, W., Chan, C., Hermens, D. F., Bennett, M. R., \& Lagopoulos, J. (2017). Frontal and subcortical grey matter reductions in PTSD. Psychiatry Research: Neuroimaging, 266, 1-9. https://doi.org/10.1016/j.pscychresns.2017.05.008

Padmanabhanunni, A., \& Edwards, D. J. (2016). Derailed by a sugar daddy: an investigation of the failed treatment of an adolescent township rape survivor with PTSD. Child Abuse Research in South Africa, 17, 83-94.

Paunović, N. (2010). Behavioral-cognitive inhibition theory: Conceptualization of post-traumatic stress disorder and other psychopathology disorders. Psychology, 1, 349-366. https://doi.org/10.4236/ psych.2010.15044

Renfrey, G., \& Spates, C. R. (1994). Eye movement desensitization: a partial dismantling study. Journal of Behavior Therapy and Experimental Psychiatry, 25, 231-239. https://doi.org/10.1016/00057916(94)90023-X

Resick, P. A., Nishith, P., Weaver, T. L., Astin, M. C., \& Feuer, C. A. (2002). A comparison of cognitiveprocessing therapy with prolonged exposure and a waiting condition for the treatment of chronic posttraumatic stress disorder in female rape victims. Journal of Consulting and Clinical Psychology, 70, 867. https://doi.org/10.1037/0022-006X.70.4.867
Rosenhan, D. L., \& Seligman, M. E. (1995). Abnormal psychology. New York: WW Norton \& Co.

Rothbaum, B. O., Cahill, S. P., Foa, E. B., Davidson, J. R., Compton, J., Connor, K. M., Astin, M. C., \& Hahn, C. G. (2006). Augmentation of sertraline with prolonged exposure in the treatment of posttraumatic stress disorder. Journal of Traumatic Stress, 19, 625-638. https://doi.org/10.1002/jts.20170

Sadatmadani, S., Tavallaii Zavareh, A., \& Tavallaii, V. (2018). Effectiveness of regular eye movement desensitization and reprocessing in therapy and reducing the symptoms of anxiety. Health Research Journal, 3, 37-43. https://doi.org/10.29252/hrjbaq.3.1.37

Scott, J. C., Harb, G., Brownlow, J. A., Greene, J., Gur, R. C., \& Ross, R. J. (2017). Verbal memory functioning moderates psychotherapy treatment response for PTSD-related nightmares. Behaviour Research and Therapy, 91, 24-32. https://doi. org/10.1016/j.brat.2017.01.004

Shapiro, F. (1994). Alternative stimuli in the use of EMD (R). Journal of Behavior Therapy and Experimental Psychiatry, 25, 89. https://doi.org/10.1016/0005-7916 (94)90071-X

Shapiro, F. (1996). Eye movement desensitization and reprocessing (EMDR): Evaluation of controlled PTSD research. Journal of Behavior Therapy and Experimental Psychiatry, 27, 209-218. https://doi. org/10.1016/S0005-7916(96)00029-8

Shapiro, F. (1998). EMDR manuals. Dhaka: Bangladesh Shapiro, F., \& Solomon, R. M. (2010). Eye movement desensitization and reprocessing. In I. Weiner \& W. E. Craighead (Eds.), The Corsini encyclopedia of psychology (4th ed., Vol. 2) (pp. 629-632). Hoboken, NJ: Wiley.

Wright, J. H., Brown, G. K., Thase, M. E., \& Basco, M. R. (2017). Learning cognitive-behavior therapy: an illustrated guide. Washington, DC: APA Publishing.

Zimmermann, P., Biesold, K. H., Barre, K., \& Lanczik, M. (2007). Long-term course of post-traumatic stress disorder (PTSD) in German soldiers: Effects of inpatient eye movement desensitization and reprocessing therapy and specific trauma characteristics in patients with non-combat-related PTSD. Military Medicine, 172, 456-460. https://doi.org/10.7205/ milmed.172.5.456
Comparing the effectiveness of EMDR and CBT on reducing $P T S D$ 